\title{
Competitive and Noncompetitive Odorant Interactions in the Early Neural Coding of Odorant Mixtures
}

\author{
Jean-Pierre Rospars, ${ }^{1}$ Petr Lansky, ${ }^{2}$ Michel Chaput, ${ }^{3}$ and Patricia Duchamp-Viret ${ }^{3}$ \\ ${ }^{1}$ Institut National de la Recherche Agronomique, Unité Mixte de Recherche (UMR) 1272, Physiologie de l'Insecte and Unité Mathématiques et Informatique \\ Appliquées, F-78000 Versailles, France, ${ }^{2}$ Institute of Physiology, Academy of Sciences of the Czech Republic, Videnska 1083, CZ-142 20 Prague 4, Czech \\ Republic, and ${ }^{3}$ Laboratoire de Neurosciences Sensorielles, Comportement, Cognition, Centre National de la Recherche Scientifique, UMR 5020, Université \\ de Lyon-Lyon 1, F-69366 Lyon Cedex 07, France
}

Most olfactory receptor neurons (ORNs) express a single type of olfactory receptor that is differentially sensitive to a wide variety of odorant molecules. The diversity of possible odorant-receptor interactions raises challenging problems for the coding of complex mixtures of many odorants, which make up the vast majority of real world odors. Pure competition, the simplest kind of interaction, arises when two or more agonists can bind to the main receptor site, which triggers receptor activation, although only one can be bound at a time. Noncompetitive effects may result from various mechanisms, including agonist binding to another site, which modifies the receptor properties at the main binding site. Here, we investigated the electrophysiological responses of rat ORNs in vivo to odorant agonists and their binary mixtures and interpreted them in the framework of a quantitative model of competitive interaction between odorants. We found that this model accounts for all concentration-response curves obtained with single odorants and for about half of those obtained with binary mixtures. In the other half, the shifts of curves along the concentration axis and the changes of maximal responses with respect to model predictions, indicate that noncompetitive interactions occur and can modulate olfactory receptors. We conclude that, because of their high frequency, the noncompetitive interactions play a major role in the neural coding of natural odorant mixtures. This finding implies that the CNS activity caused by mixtures will not be easily analyzed into components, and that mixture responses will be difficult to generalize across concentration.

Key words: sensory coding; olfactory receptor neuron; in vivo; signal transduction; allostery; mixture interaction

\section{Introduction}

Many behaviors in invertebrates and vertebrates, including feeding, reproductive, and social behaviors, depend on, to a large extent, olfaction. The odorous environment of terrestrial animals is extremely rich, because the number of volatile organic molecules is large and also because they combine in various amounts to create a virtually infinite number of blends. Most odors present in the environment are these complex mixtures consisting of dozens, often hundreds of components, and olfactory systems have evolved to recognize and discriminate them (Laing and Francis, 1989; Laska and Hudson, 1992; Derby et al., 1996). The initial event of the sensory process consists in the interaction of odorant molecules with an array of G-protein-coupled odorant receptors (ORs) borne by the dendritic membrane of olfactory receptor neurons (ORNs) (Buck and Axel, 1991; Godfrey et al.,

Received July 30, 2007; revised Dec. 17, 2007; accepted Jan. 20, 2008.

This work was supported by Agence Nationale de la Recherche Grant ANR-05-PNRA-1.E7 Aromalim (J.-P.R., P.D.-V., M.C.), Ministère des Affaires étrangères Grant EC0-NET 12644PF (J.-P.R., P.L.), Grant Barrande 09146 QL between Czech Republic and France (J.-P.R., P.L.), Grant AV0Z50110509, Centre for Neurosciences Grant LC554, and Information Society Grant 1ET4000110401 (P.L.). We thank Drs. L. Kostal, T. Thomas-Danguin, E. Pajot, G. Sanz, C. Gadenne, P. Lucas, S. Anton, and C. Young and three anonymous referees for helpful advice.

Correspondence should be addressed to Dr. Jean-Pierre Rospars, Institut National de la Recherche Agronomique, Unité Mixte de Recherche 1272, Physiologie de I'Insecte, F-78000 Versailles, France. E-mail: rospars@versailles.inra.fr.

DOI:10.1523/JNEUROSCI.4670-07.2008

Copyright $\odot 2008$ Society for Neuroscience $\quad$ 0270-6474/08/282659-08\$15.00/0
2004; Malnic et al., 2004). With a few exceptions, each ORN expresses only one of their OR genes. Each OR can recognize several odorants, and each odorant can be recognized by more than one OR, each odorant-OR interaction being characterized by a specific affinity (Araneda et al., 2004; Sanz et al., 2005).

Various behavioral, psychophysical, and neurophysiological experiments have shown that the response to an odorant mixture is not a simple function of the responses to its individual components, a phenomenon called mixture interaction (Laing et al., 1989). Mixture suppression (Gleeson and Ache, 1985), where the response to a mixture is less than that to either of its components alone, is far more common than mixture enhancement (Kang and Caprio, 1997). Duchamp-Viret et al. (2003) in rat ORNs distinguished several types of interactions depending on whether the firing response to a binary mixture is less than the least effective component (inhibition) and less (suppression), equivalent but not higher (hypoadditivity) or higher (synergy), than the most effective component.

Competitive antagonism between two odorants (Oka et al., 2004b; Sanz et al., 2005) and suppression elicited by agonists (Sanz et al., 2005) were recently demonstrated using ORs expressed in heterologous systems. Here, we investigated whether the mere competition of two agonists for the same receptor site (syntopic interaction) (Neubig et al., 2003) could account for mixture interaction. We developed a model of competitive (syn- 
topic) interaction where agonists first bind to the receptor and then activate it. The model predicts the concentrationresponse curve of a binary mixture knowing those of its components. We used it as a reference to analyze microelectrode recordings from rat ORNs. We showed that it accounts for single odorant curves and can explain the hypoadditive and suppressive effects. It predicts accurately the position along the concentration axis (apparent affinity, related to binding) and maximum response (efficacy, related to activation) of approximately half the mixture curves. However, its predictions were not validated in the other mixture curves. In theses cases, an odorant can increase or decrease the affinity and/or efficacy of another odorant, these four effects being equiprobable. These noncompetitive interactions, which account for the observed synergies, can modify to a considerable extent the qualitative code of a mixture with respect to the codes of its components.

\section{Materials and Methods}

Animal preparation and recordings. Rats were recorded as before (Duchamp-Viret et al., 2000, 2003). Briefly, adult Wistar rats were deeply anesthetized with an injection of equithesin and then tracheotomized and secured in a stereotaxic apparatus. Recordings were performed in the Endoturbinate II. Nasal bones were removed, and the dorsal recess underlying them was slipped aside. Single-unit action potentials were recorded using metal-filled glass micropipettes (2-4 M $\Omega$ ). The constancy of the shape of the recorded spikes was checked during the experiments to ensure that the same single ORN was recorded. Responses were shown to be reproducible for the same stimulus at the same concentration (see below) and as not displaying any decrement over time during recording.

Odor stimulations. The 10 odorants tested (Table 1) were obtained in the highest purity available from Sigma Aldrich, except lilial (LIL) (IFF Global Headquarters, New York, NY) and isomenthone (MEN) (Interchim, Montluçon, France). Racemic mixtures of enantiomers were used. The concentrations of the saturating vapors (SVs) were derived from the SV pressures $P_{0}$ as given by the program MBPwin of the EPIsuite 3.12 software (United States Environmental Protection Agency).

For each recorded ORN, the odorants were tested at a concentration close to SV, and the most effective were selected for establishing their dose-response curves. Odorants were delivered using a dynamic flow multistage olfactometer (Vigouroux et al., 1988) as $2 \mathrm{~s}$ square pulses, with precisely controlled concentrations and time course, applied directly near the surface of the olfactory mucosa at a constant temperature $\left(22^{\circ} \mathrm{C}\right)$ and flow rate $(200 \mathrm{ml} / \mathrm{min})$. Twelve dilutions of the $\mathrm{SV}$ were available, the dilution factors being $d=2 \times 10^{i / 4}$, where $i$ are integers from $0(d=2)$ to $11(d=1125)$. The concentrations of single odorants $\mathrm{U}$ and $\mathrm{V}$ were $\mathrm{U}=U_{0} / d$ and $V=V_{0} / d$, where $U_{0}$ and $V_{0}$ are the molarities of their SVs. Dilutions and mixing of binary mixtures $\mathrm{U}+\mathrm{V}$ were done in the air phase. The same dilution $d$ was used for both $\mathrm{U}$ and $\mathrm{V}$, so the mixture concentration was $M=\left(U_{0}+V_{0}\right) / d$. The same number of molecules $\mathrm{U}$ (respectively $\mathrm{V}$ ) was present in the mixture and the single-odorant stimulus $\mathrm{U}$ (respectively $\mathrm{V}$ ) at the same dilution. Thus, the total number of molecules was different in equal volumes of the stimuli $\mathrm{U}, \mathrm{V}$, and $\mathrm{U}+\mathrm{V}$, but the ratio $r=U / V=U_{0} / V_{0}$ remained constant at different dilutions. The dose-response curves were established in the order odorant $U$, mixture $U+V$, and odorant $V$. For each odor, the responses were recorded
LIM
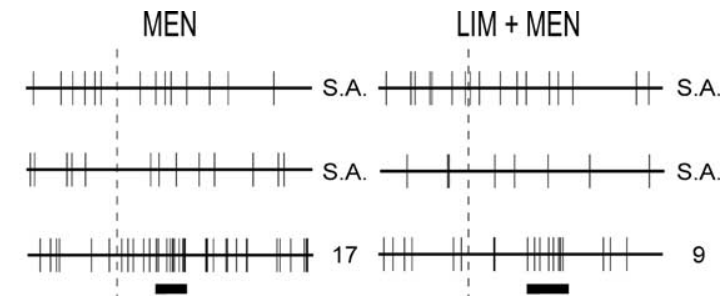

9
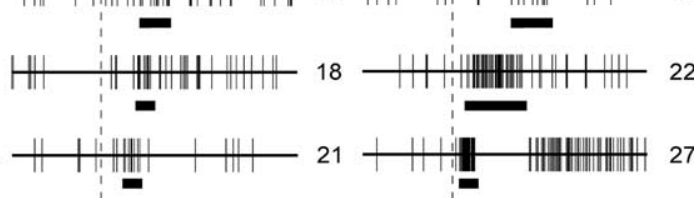

27
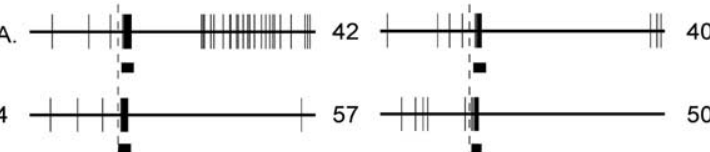

17

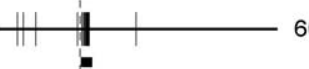

60
61

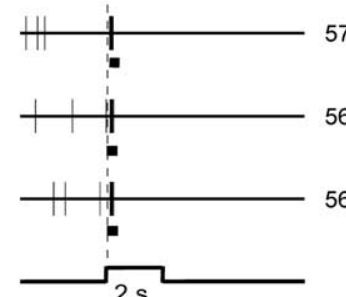

$2 \mathrm{~s}$

Figure 1. Examples of spontaneous and evoked activity recorded from the same ORN (37.6) at increasing concentrations of (Stim). The deturating vapor is given on the left. The dashedvertical line indicatesthe beginning second, are given on the right of spike trains.

at increasing concentrations. At least five concentrations of each stimulus were tested from close to threshold to close to SV. In each stimulation sequence, the cell activity was recorded $30 \mathrm{~s}$ before and $30 \mathrm{~s}$ after a $2 \mathrm{~s}$ odor pulse delivery. Successive stimulations with the same odor were separated by at least $2 \mathrm{~min}$, and a $5 \mathrm{~min}$ rinsing period was maintained between two different odors. More than $1 \mathrm{~h}$ was needed to establish the concentration-response curves of a complete set of odors ( $\mathrm{U}, \mathrm{V}$, and $\mathrm{U}+$ $\mathrm{V})$ with a single trial per concentration. Priority was given to testing more odors per ORN over repeated trials of the same stimulus at the same concentration.

Quantitative analyses of responses and dose-response curves. Responses in recorded spike trains (Fig. 1) were determined as follows (DuchampViret et al., 1989). The $m$ interspike intervals in the $30 \mathrm{~s}$ prestimulus period were compared with all uninterrupted sequences, which could be formed from the $n$ poststimulus intervals included in a $12 \mathrm{~s}$ time period after the onset of the stimulus. These sequences were all possible groups of successive poststimulus intervals, starting at the first, second,. . . or $n$th interval and including $1,2, \ldots$ or at most $n$ intervals (i.e., starting with the $i$ th interval, we took the next $j$ intervals, as long as $i+j \geq n$ ). Then, we tested whether the collection of $j$ poststimulus intervals, of each of the $n(n$ - 1)/2 sequences with at least two intervals, was statistically distinguishable from the $m$ prestimulus intervals using the nonparametric MannWhitney test at level 5\%. All sequences significantly different from the prestimulus intervals were determined and, among them, those having the shortest latency (and sharing for this reason the same first spike) were selected. The longest of these sequences was considered as the response.

The response frequency was defined as the mean of the instantaneous firing rates (inverses of the interspike intervals) in the response. This method is robust and gives practically the same results as the different algorithm presented by Rospars et al. (2003). We also observed, in these and other experiments, that repeated stimulations with the same ORN, odor, and concentration yielded firing frequencies that varied by $<15 \%$. Although, like in amphibians (Reisert and Matthews, 1999; Rospars et al., 2003), the latency, duration, and frequency of responses were strongly dependent on odor concentration, the interspike intervals in the same 
Table 1. Sample of odorants used

\begin{tabular}{llllll}
\hline Abb. $^{a}$ & CAS number $^{b}$ & Name & Formula & $P(\mathrm{mmHg})^{c}$ & $M_{0}(\mathrm{~mol} / \mathrm{L})^{d}$ \\
\hline EVA & $121-32-4$ & Ethylvanillin & $\mathrm{C}_{9} \mathrm{H}_{10} \mathrm{O}_{3}$ & $1.04 \times 10^{-5}$ & $5.60 \times 10^{-10}$ \\
LYR & $31906-04-4$ & Lyral & $\mathrm{C}_{13} \mathrm{H}_{22} \mathrm{O}_{2}$ & $2.73 \times 10^{-5}$ & $1.46 \times 10^{-9}$ \\
LIL & $80-54-6$ & Lilial & $\mathrm{C}_{14} \mathrm{H}_{20} 0^{-}$ & 0.00360 & $1.93 \times 10^{-7}$ \\
CAM & $76-22-2$ & Camphor & $\mathrm{C}_{10} \mathrm{H}_{16} \mathrm{O}$ & 0.0720 & $3.87 \times 10^{-6}$ \\
CIT & $5146-66-7$ & Citralva & $\mathrm{C}_{10} \mathrm{H}_{15} \mathrm{~N}$ & 0.0362 & $1.95 \times 10^{-6}$ \\
MEN & $18309-28-9$ & Isomenthone & $\mathrm{C}_{10} \mathrm{H}_{18} \mathrm{O}$ & 0.370 & $1.99 \times 10^{-5}$ \\
LIM & $138-86-3$ & Limonene & $\mathrm{C}_{10} \mathrm{H}_{16}$ & 1.45 & $7.80 \times 10^{-5}$ \\
ANI & $100-66-3$ & Anisole & $\mathrm{C}_{7} \mathrm{H}_{8} \mathrm{O}$ & 3.54 & $1.90 \times 10^{-4}$ \\
MAK & $110-43-0$ & Methyl-amyl-ketone & $\mathrm{C}_{7} \mathrm{H}_{14} 0$ & 3.86 & $2.08 \times 10^{-4}$ \\
ISO & $123-92-2$ & Isoamyl acetate & $\mathrm{C}_{7} \mathrm{H}_{14} 0_{2}$ & 5.60 & $3.01 \times 10^{-4}$ \\
\hline
\end{tabular}

${ }^{a}$ Abbreviated names used in this study.

${ }^{b}$ Chemical abstract numbers.

'Saturating vapor pressures at $T=298 \mathrm{~K}$ as given in EPIsuite (see Materials and Methods).

${ }^{d} M_{0}$, Molarity of saturating vapor; $M_{0}=P / R T$ with $r=62.364 \mathrm{~L} . \mathrm{mmHg} \cdot \mathrm{K}^{-1} \cdot \mathrm{mol}^{-1}$.

response were usually of similar lengths (Fig. 1). The intervals gave no indication of a systematic variation in time and were thus compatible with the following steady-state description of the responses.

We interpreted the responses to single odorants in the framework of a model originally proposed by Del Castillo and Katz (1957) involving a two-step OR activation, which is the simplest model accounting for variations in affinity and efficacy between different agonists (Rang, 2006). In the first step (association), the odorant binds to (or unbinds from) the OR. In the second step (activation), the odorant-OR complex undergoes a transition to (or from) an activated state (Rospars et al., 1996, 2003). Then, assuming that the following transduction steps depend only on the number of activated ORs and the properties of the cascade, it can be shown (supplemental note, available at www.jneurosci.org as supplemental material) that the firing rate is a so-called Hill function of odorant concentration (Eqs. A4-A5 in supplemental note, available at www. jneurosci.org as supplemental material). This is a sigmoid curve characterized by the following: (1) its asymptotic maximal firing rate $F_{\mathrm{M}} ;(2)$ its position along the dose axis, given by the odorant concentration $K$ that produces $50 \%$ of the maximal firing (abbreviated $\mathrm{EC}_{50}$ ); and (3) its slope, given by Hill coefficient $n$. K is controlled primarily by the association step and $F_{\mathrm{M}}$ by the activation step, whereas $n$ is independent of the odorant-OR interaction and constant for a given ORN.

Statistical model testing. The parameters of the model were determined in two steps. First, Hill coefficients were determined for all curves available. The hypothesis that they are equal for a given ORN was tested using Holm-adjusted $t$ tests (Aickin and Gensler, 1996), and a common estimate of $n$ per ORN was calculated. Second, using the common $n$ values, the two other parameters [i.e., position $\mathrm{EC}_{50}$ values $\left(K_{\mathrm{U}}, K_{\mathrm{V}}\right.$ for single odorants, $K_{\mathrm{o}}$ for their mixtures) and asymptotic maxima $\left(F_{\mathrm{MU}}, F_{\mathrm{MV}}\right.$, and $\left.F_{\mathrm{Mo}}\right)$ ] were estimated using the nlinfit function of the Matlab Statistics Toolbox (MathWorks, Natick, MA).

The predicted curve for the mixture was calculated with Equation 1 in which parameters $K_{\mathrm{p}}$ and $F_{\mathrm{Mp}}$ were calculated using Equations 2 and 3 knowing the parameters $\left(K_{\mathrm{U}}, K_{\mathrm{V}}, F_{\mathrm{MU}}, F_{\mathrm{MV}}, n\right)$ determined above. For comparing the observed characteristics $\left(K_{\mathrm{o}}, F_{\mathrm{Mo}}\right)$ of each mixture to the predicted ones $\left(K_{\mathrm{p}}, F_{\mathrm{Mp}}\right)$ and testing the hypothesis of syntopic interaction, we studied separately the $\mathrm{EC}_{50}$ values and the maxima.

Each pair of $\mathrm{EC}_{50}$ values $\left(\log K_{\mathrm{o}}, \log K_{\mathrm{p}}\right)$ was compared using a $t$ test. The SDs, $\sigma_{\mathrm{o}}$ and $\sigma_{\mathrm{p}}$, of these two quantities were calculated as follows. First, for each set of Hill curves, the half- $95 \%$ confidence intervals of the fitted parameters $\left(\Delta \log K_{\mathrm{U}}, \Delta \log K_{\mathrm{V}}\right.$ for single odorants, $\Delta \log K_{\mathrm{o}}$ for mixtures, and $\Delta n$ for a given ORN) were calculated using the nlparci function of the Matlab Statistics Toolbox. Second, for each mixture, the uncertainty $\Delta \log K_{\mathrm{p}}$ on the predicted $\mathrm{EC}_{50}$ was derived from $\Delta \log K_{\mathrm{U}}$, $\Delta \log K_{\mathrm{V}}$, and $\Delta n$ using the standard procedure for uncertainty calculations, as explained in supplemental note section 5 (available at www. jneurosci.org as supplemental material). Third, the SDs $\sigma_{\mathrm{o}}$ of $\log K_{\mathrm{o}}$ and $\sigma_{\mathrm{p}}$ of $\log K_{\mathrm{p}}$ were calculated from their half- $95 \%$ confidence intervals $\Delta \log K_{\mathrm{o}}$ and $\Delta \log K_{\mathrm{p}}$ using the relationship $\sigma=\Delta \sqrt{ } N / 1.96$. To reach a global conclusion based on a series of $v$ independent $t$ statistics, we cal- culated $S=\sum_{i=1}^{\theta}-2 \ln \left(1-P_{i}\right)$, where $P_{i}$ is the significance level of the $i$ th $t$ statistic. Quantity $S$ is a $\chi^{2}$ random variable with $2 v$ degrees of freedom if observed and predicted characteristics are the same. If $S \geq \chi^{2}(1-\alpha, 2 v)$, the combined weight of all $t$ tests is sufficient to reject $H_{0}$, with probability of type I error equal to $\alpha$ [Larson, 1973 (p. 132)]. For having independent $t$ tests, only one curve per ORN was selected (nine tests in all) while keeping the same proportion of significant and nonsignificant differences as in the original sample (15 sets).

The same procedure was followed for testing the equality of asymptotic maxima $F_{\mathrm{Mo}}$ and $F_{\mathrm{Mp}}$.

\section{Results \\ Global characteristics of dose-response curves}

The activity of 133 ORNs in response to a varied set of odorants (Table 1) and their binary mixtures at several concentrations were recorded in vivo, and their firing rates were determined (Fig. $1)$. The necessary conditions for a complete quantitative analysis were met by 68 dose-response curves from 21 neurons (Table 2). These curves (1) include at least five concentrations, (2) present a well-defined threshold, and (3) explore the whole dynamic range up to a discernible asymptote at a dose close to saturating vapor. In practice, the first two conditions were the most selective. Relaxing the third condition resulted in the addition of only a few curves, which made application of the near-threshold approximation (see section 4 of the supplemental note, available at www.jneurosci.org as supplemental material) not worth doing to test the syntopic model.

All 68 dose-response curves presented a sigmoid shape. We verified that they were accurately described by Hill function (see Materials and Methods) with the asymptotic maximum $F_{\mathrm{M}}$ and the concentration $K$ at half-maximum response, but not the Hill coefficient $n$, depending strongly on the odor (Fig. 2). Each odor tested, whether a single odorant or a mixture, yielded a different $\mathrm{EC}_{50}$ value and maximum, the largest contrasts in the same cell being $10^{-10.3}$ to $10^{-5.5} \mathrm{M}$ for $\mathrm{EC}_{50}$ values and $6-51$ spikes/s for maxima. For all odor-ORN pairs together, the $\mathrm{EC}_{50}$ values were in the range of $10^{-13.5}$ to $10^{-4.3} \mathrm{M}$ (Fig. $3 A$ ) and the maximal firing rates in the range of $6-218$ spike/s (Fig. $3 B$ ).

\section{Response characteristics of single odorants and binary mixtures in complete sets}

Only complete sets, including at least two single odorants and their mixture, can be used for testing the syntopic model. This stringent fourth condition left a subsample of 15 complete sets, including 38 curves recorded from nine neurons (Table 2). We checked that these nine neurons were truly representative of the sample of 21 neurons by comparing all three characteristics, $K$, $F_{\mathrm{M}}$, and $n$, of the curves from the complete sets with those from the incomplete sets (Fig. $3 A-C$ ). Kolmogorov-Smirnov tests showed that their distributions were not statistically different, supporting the conclusion that the curves in both categories did not differ in any significant way. All other results below are based only on the 38 curves from the complete sets.

Second, we showed that the distributions of $\mathrm{EC}_{50}$ values (Fig. $4 A$ ) and maxima (Fig. $4 B$ ) of both single odorants and mixtures in complete sets were very similar, except for a slight (nonsignificant) difference for the curves with small $\mathrm{EC}_{50}$ values. This means that, qualitatively and quantitatively, a dose-response 
Table 2. Available data

\begin{tabular}{lllll}
\hline Category & Rats & Neurons & Sets & Curves \\
\hline Total & 40 & 133 & & \\
Sets with at least one mixture curve $^{a}$ & 31 & 40 & 55 & 149 \\
Incomplete sets $^{b}$ of curves & 14 & 21 & 27 & 75 \\
Complete curves $^{c}$ from incomplete sets & 13 & 14 & 18 & 30 \\
Complete sets $^{d}$ of curves $(U, V, U+V)$ & 17 & 19 & 28 & 74 \\
Complete curves from complete sets $^{\text {Com }}$ & 8 & 9 & 15 & 38 \\
\hline
\end{tabular}

${ }^{a} \mathrm{~A}$ "curve" is a concentration-response plot of at least five points (plots with less points were not analyzed).

${ }^{b}$ An incomplete set includes only one or two of the three curves $(U, V, U+V)$.

In a complete curve, both threshold and asymptote are known.

${ }^{d} \mathrm{~A}$ complete set includes curves for both single odorants $\mathrm{U}$ and $\mathrm{V}$ and their mixture $\mathrm{U}+\mathrm{V}$.

curve evoked by a binary mixture cannot be distinguished from one evoked by a single odorant.

Third, in most curves studied, the frequencies yielded by the two highest concentrations tested were practically equal, or displayed a clearly noticeable change in the slope of the curve, indicating their closeness to an asymptote. This property is important, because this is the only reliable way to assign both $F_{\mathrm{M}}$ and $K$.

Fourth, we checked that, in general, this observed asymptotic firing could not result from some limiting step in the transduction cascade or the action-potential generator, and so reflected accurately OR activation. Take, for example, ORN 15.4 for which five curves are available involving stimulations with anisole (ANI), isoamyl acetate (ISO), and methyl-amyl-ketone (MAK). Their maxima $F_{M}$ were 150, 150, 153, 192, and 218 spikes/s for ANI, ISO, ANI+ISO, ANI+MAK, and MAK, respectively. For the highest frequency recorded in this cell $\left(F_{\max }=218\right)$, electrical saturation cannot be decided. However, any $F_{\mathrm{M}}$ less than, for example, $F_{\max } / 1.2=182$ can be considered as not resulting from electrical saturation, because it could have fired at a frequency at least $20 \%$ greater. Three of the maxima are below the limit, and only one (ANI+MAK) may possibly be saturated. The same procedure was applied to all neurons. On 29 testable curves (38 curves minus the $9 F_{\max }$ values for which no check was possible), 23 (i.e., 79\%) were found to be below the limits $F_{\max } / 1.2$. So the majority of curves gave no indication of electrical saturation.

\section{Model of syntopic interaction}

Two odorants, A and B, applied separately to the same ORN will usually give two Hill curves with different maxima $\left(F_{\mathrm{MU}}, F_{\mathrm{MV}}\right)$ and $\mathrm{EC}_{50}$ values $\left(K_{\mathrm{U}}, K_{\mathrm{V}}\right)$, which may intersect (Fig. $5 A$ ) or not (Fig. $5 B$ ). When they are applied together as a binary mixture $\mathrm{U}+\mathrm{V}$, the two agonists compete for the available OR sites, because each OR can be occupied by a single molecule, $U$ or $V$. If the binding of $U$ to the $\mathrm{OR}$ and the subsequent $\mathrm{OR}$ activation are not modified by the presence of $\mathrm{V}$, and vice versa [i.e., if only competitive (syntopic) interaction is present, without any change of the rate constants and therefore of the parameters $K_{\mathrm{U}}, K_{\mathrm{V}}, F_{\mathrm{MU}}$, and $F_{\mathrm{MV}}$ in the mixture], the OR response to $\mathrm{U}+\mathrm{V}$ can be determined from chemical kinetics. Then, with no change of the transduction cascade, the ORN response to the mixture can be derived from its responses to the single components. In the case where the ratio $U / V$ is a constant $r$ at any dose, as in our experiments, we showed (supplemental note section 3, available at www. jneurosci.org as supplemental material) that the spiking response to the mixture is also a Hill function of the dose $M=U+V$ :

$$
F_{\mathrm{U}+\mathrm{V}}(M)=\frac{F_{\mathrm{Mp}}}{1+\left(\frac{K_{\mathrm{p}}}{M}\right)^{n}},
$$

where the $\mathrm{EC}_{50}, K_{\mathrm{p}}$, and maximum, $F_{\mathrm{Mp}}$ (subscript "p" is "predicted"), are given by the following:

$$
\begin{gathered}
K_{\mathrm{P}}=(1+r) K_{\mathrm{U}} K_{\mathrm{V}} \sqrt[n]{\frac{1}{r^{n} K_{\mathrm{V}}^{n}+K_{\mathrm{UA}}{ }^{n}}} \\
F_{\mathrm{Mp}}=\frac{F_{\mathrm{MU}} r^{n} K_{\mathrm{V}}^{n}+F_{\mathrm{MV}} K_{\mathrm{U}}^{n}}{K_{\mathrm{U}}^{n}+r^{n} K_{\mathrm{V}}^{n}} .
\end{gathered}
$$

$F_{\mathrm{Mp}}$ depends on all six parameters, whereas $K_{\mathrm{p}}$ depends on only four, being independent of the maxima $F_{\mathrm{MU}}$ and $F_{\mathrm{MV}}$. The $\mathrm{EC}_{50}$ of the mixture can be shown to lie between the $\mathrm{EC}_{50}$ values of its single components, and the maximum between their maxima. Whatever the concentration, the response curve to the mixture is intermediate between the response curves to the components. As shown in Figure 5, its exact position depends on the respective values of the parameters and especially of $r$.

\section{Comparison of predicted and observed responses to mixtures} Qualitative comparisons of the experimental curves for mixtures to those of their components revealed noteworthy effects. First, some of the observed mixture curves were located between the component curves (Fig. $2 A, B$ ) and were therefore compatible with the syntopic model. However, in these cases, especially when the apparent affinities were very different, for example in Figure $2 A$, the odorant with the least affinity [limonene (LIM)] had a profound effect, even at concentrations well below its apparent threshold, on the responses to the other odorant (MEN). Whether this effect was attributable to competitive or noncompetitive interaction cannot be resolved by mere comparison of the experimental data. Second, other curves were not between the component curves. For example, the mixture curve can be below the lowest component curve (Fig. $2 C$, suppression) or above the highest curve on part of the response range (Fig. 2 D, synergy). A single mixture curve was found completely below the lowest component curve (full range suppression) (data not shown), although none was found completely above (full synergy). These examples suggest that syntopic interaction was not the rule but raise the question of their statistical significance.

Thus, qualitative examination of the curves is not sufficient to reach definite conclusions. To analyze the origin of the observed effects, we calculated with Equations 2 and 3 the predicted syntopic response characteristics $\left(K_{\mathrm{p}}, F_{\mathrm{Mp}}\right)$ of each mixture tested from the observed characteristics $\left(K_{\mathrm{U}}, F_{\mathrm{MU}}, K_{\mathrm{V}}, F_{\mathrm{MV}}\right)$ of their single components, and we compared them to their observed counterparts $\left(K_{\mathrm{o}}, F_{\mathrm{Mo}}\right)$ obtained by direct fitting of the mixture data. As shown in Table 3 , the differences in position $\log K_{\mathrm{o}}-\log$ $K_{\mathrm{p}}$ varied from 0.02 to $0.62 \log$ units (i.e., the ratios $K_{\mathrm{o}} / K_{\mathrm{p}}$ varied from 1 to 4.2 ), and the differences in asymptotic maxima $F_{\mathrm{Mo}}-$ $F_{\mathrm{Mp}}$ varied from 1.1 to 35.5 spikes/s. Because the syntopic model predicts no difference, we tested the statistical significance of each difference against zero by $t$ tests.

In $47 \%$ of comparisons (Table 3 ), the Hill curve fitted to the experimental data were undistinguishable from the reference curve predicted by the syntopic model, including the example of Figure $2 \mathrm{~A}$ described above. Although profound, the strong subthreshold effect can be fully accounted for by the syntopic model, all experimental points for LIM + MEN being very close to the theoretical curve given by Equations 2 and 3 (Fig. 6A).

In the remaining cases, the observed Hill curves significantly departed from the reference curves in $\mathrm{EC}_{50}$, maximum, or both $\mathrm{EC}_{50}$ and maximum. The meta-analyses of independent $t$ tests yielded statistics $S=40.11\left(p=2.02 \times 10^{-3}\right)$ for $\mathrm{EC}_{50}$ values and 

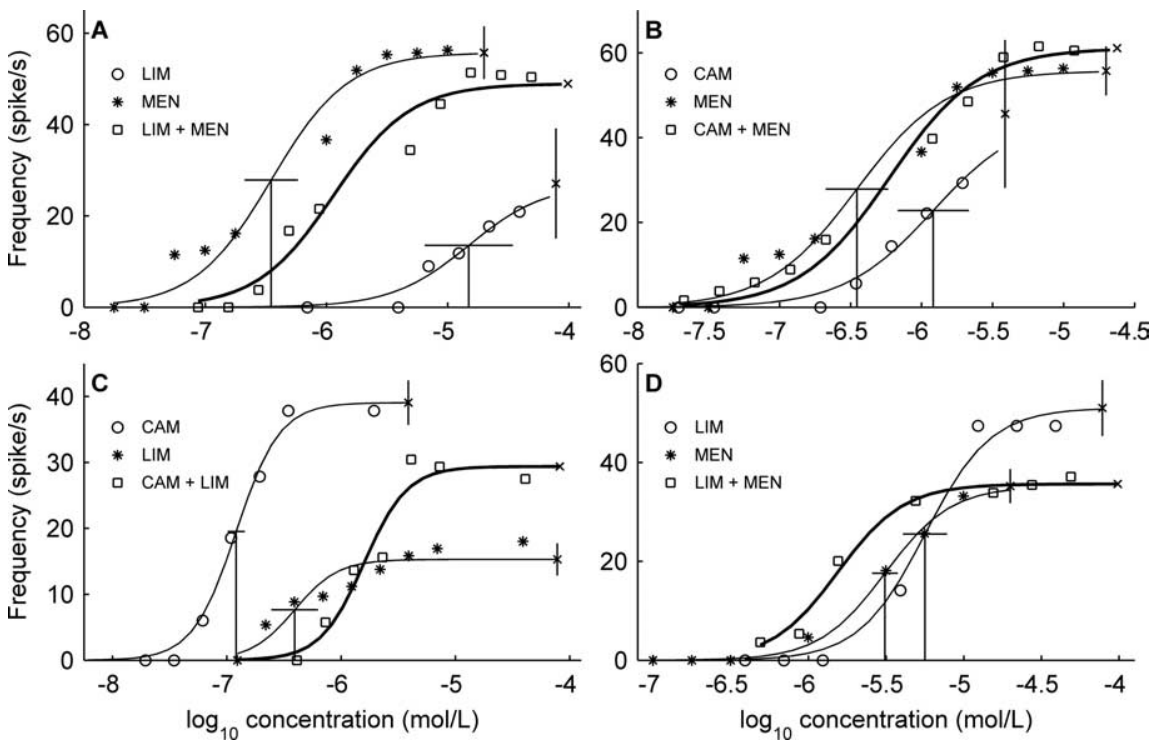

Figure 2. Examples of sets of dose-response curves comparing the firing rates measured in response to single odorants (circles, asterisks, thin lines) and their binary mixture (squares, thick lines) in ORN 37.6 $(\boldsymbol{A}, \boldsymbol{B}), 17.3(\boldsymbol{C})$, and 17.1 (D). Hill curves were fitted to experimental points. $\mathrm{EC}_{50}$ values (logK, vertical line at midrange) with their $95 \%$ confidence intervals ( $\pm \Delta \log K$, horizontal segment on top) and maximal responses $\left(F_{M}\right)$ with $95 \%$ confidence interval ( $\pm \Delta F_{M}$, vertical segment) are shown for single odorants (for mixtures, see Figs. 4, 5). The cross at the end of each curve indicates both the concentration of the saturating vapor and the estimated asymptotic maximum $F_{M}$. Suppression (squares between asterisks and circles at all concentrations in $\boldsymbol{A}$, below $10^{-5.8} \mathrm{M} \mathrm{in} \boldsymbol{C}$, and above $10^{-5.2} \mathrm{M}$ in $\boldsymbol{D}$ ), hypoadditivity (squares close to asterisks in $\boldsymbol{B}$ ), inhibition above $10^{-5.8} \mathrm{M}$ (squares below asterisks; $\boldsymbol{C}$, and synergy below $10^{-5.2} \mathrm{M}$ (squares above asterisks; $\boldsymbol{D}$ ).
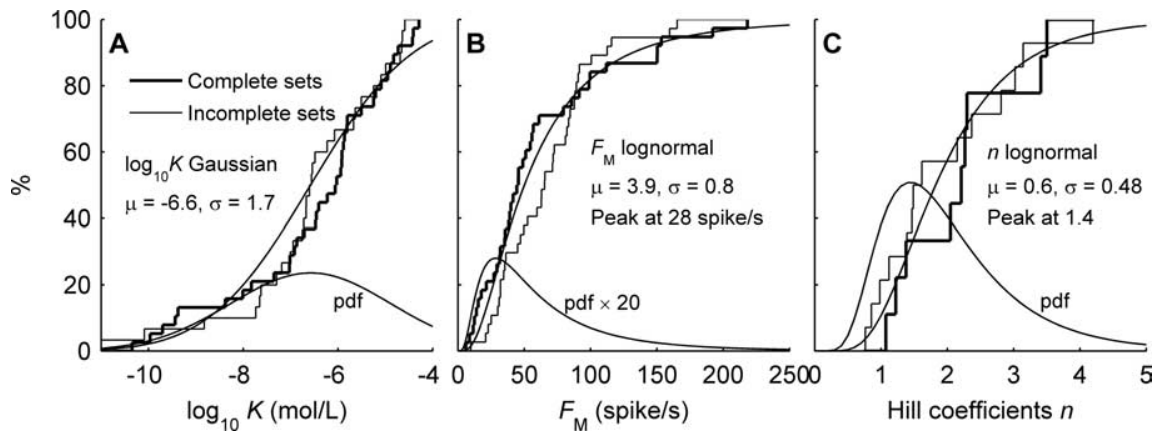

Figure 3. Cumulative histograms of $\mathrm{EC}_{50}$ values $(\boldsymbol{A})$, asymptotic firing rates $(\boldsymbol{B})$, and Hill coefficients $(\boldsymbol{C})$. For any value chosen on the horizontal axis, the stair-like function indicates the proportion of dose-response curves with an $\mathrm{EC}_{50}$, asymptote, or Hill coefficient smaller than this value. Response characteristics in complete sets (in which at least two single odorants and their mixture were tested; thick stair-like lines) and incomplete sets (in which a single odorant and/or the mixture is missing; thin stair-like lines) are not significantly different as shown by Kolmogorov-Smirnov tests ( $p$ values: 0.29 for $\log K, 0.09$ for $F_{M}$, and 0.87 for $n$ ). Cumulative probability distributions (thin smooth curves) and corresponding probability density functions (thin lines denoted "pdf"), fitted to data from both complete and incomplete sets together, are Gaussian $(\boldsymbol{A})$ and lognormal $(\boldsymbol{B}, \boldsymbol{C})$ (i.e., log $F_{\mathrm{M}}$ and $\log n$ are Gaussian). In $\boldsymbol{A}, \mu$ and $\sigma$ are the mean and $S D$ of $\log _{10} K ;$ in $\boldsymbol{B}$ and $\boldsymbol{C}$, they are the parameters of the lognormal distribution [i.e., the mean and SD of $F$ and $n$ are $\exp (\mu)$ and $\exp (\sigma)$, respectively].

$S=60.93\left(p=1.44 \times 10^{-6}\right)$ for maxima, showing that the combined evidence for rejecting the syntopic model is very significant. The cases with significant differences did not show any preferred direction, the observed curves being shifted in almost equal numbers to the right (2), to the left (3), above (3), and below (3) the reference curves (Fig. 7, arrows). However, the mean deviations regardless of direction were relatively large, with $0.44 \log$ units for $\mathrm{EC}_{50}$ values and 19 spikes/s for asymptotes, which represent $35 \%$ of the dynamic ranges of observed mixture curves and more than half of their maximum frequencies, respectively.

\section{Discussion}

Observed dose-response relationships

The main question addressed here is whether the neural coding of mixtures of odorant molecules by ORNs can be predicted from a proper knowledge of the neural coding of their components. Answering this question requires that the dose-response curves of individual rat ORNs can be described quantitatively. We showed that this is the case. As in amphibians (Firestein et al., 1993; Reisert and Matthews, 1999; Rospars et al., 2003), the response curves to single odorants are all well described by Hill functions. This is also true for mixtures $\mathrm{U}+\mathrm{V}$ in which the ratio $U / V$ of the concentrations of the components at any total concentration $U+V$ is constant. So, the dose-response curves for all stimuli were described in the same way. Depending on the stimulus, the curves from the same ORN differed in their $\mathrm{EC}_{50}$ and their maximum firing rate. Like in the frog (Rospars et al., 2003), the distributions of the logarithms of the $\mathrm{EC}_{50}$ values (Fig. 3A) and maxima (Fig. 3B) are Gaussian, but the mean and variance of the maxima are approximately twice as large as in the frog. Also, like in the frog (Rospars et al., 2003) and mouse (Grosmaittre et al., 2006), the dynamic range, which depends on the Hill coefficient (Fig. 3C), can be very broad and exceed 1000-fold of odorant concentrations from threshold to saturation.

These properties can be quantitatively interpreted in the framework of a transduction model in which the most important step is the initial odorant-OR interaction. To apply the model, each ORN must express a single type of OR, which is considered as the rule in mammals (Serizawa et al., 2003). Two other related conditions are required: the concentration of activated ORs must reach an asymptotic maximum for a large enough stimulus concentration, smaller than or equal to the saturating vapor concentration, and all postreceptor transduction steps involved in receptor potential and spike train generations, must not be limiting. The data at hand were in good agreement with these conditions, because in most curves, the asymptotic tendency was visible and most asymptotic maxima were much smaller than the largest firing rate observed in the same ORN. In short, the maximum firing frequency was generally the consequence of activated ORs reaching their asymptotic maximum. The fact that different odorants yielded different maxima in the same ORN indicates that odorants are partial agonists of the ORs, which is consistent with the analysis of series of molecules interacting with the I7 receptor (Araneda et al., 2000) and two human olfactory receptors (Sanz et al., 2005). In our model, this variation in effi- 

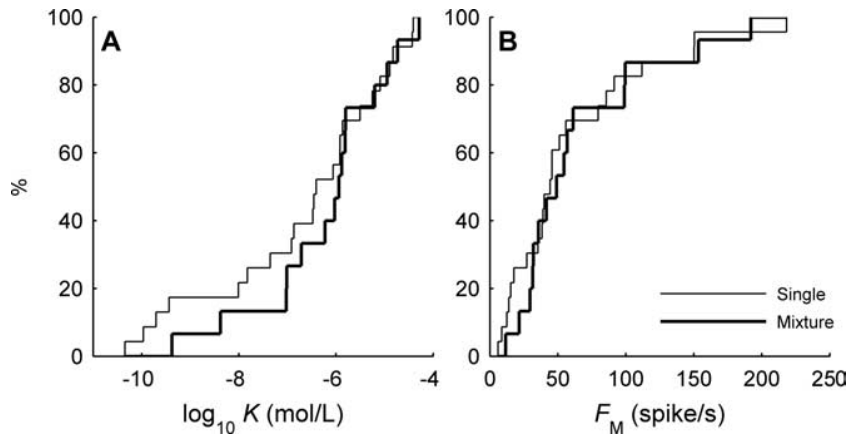

Figure 4. Comparison of $\mathrm{EC}_{50}$ values $(\boldsymbol{A})$ and asymptotic firing rates $(\boldsymbol{B})$ for single odorants (thin lines, $K_{\mathrm{U}}, K_{\mathrm{V}}$ and $F_{\mathrm{MU}}, F_{\mathrm{MV}}$ ) and binary mixtures (thick lines, $K_{0}, F_{\mathrm{Mo}}$ ) in complete sets. Same representation as in Figure 3 . Distributions of response characteristics for single odorants and mixtures are not significantly different (Kolmogorov-Smirnov tests; $p$ values, 0.87 for $\log K$, 0.84 for $F_{M}$ ).
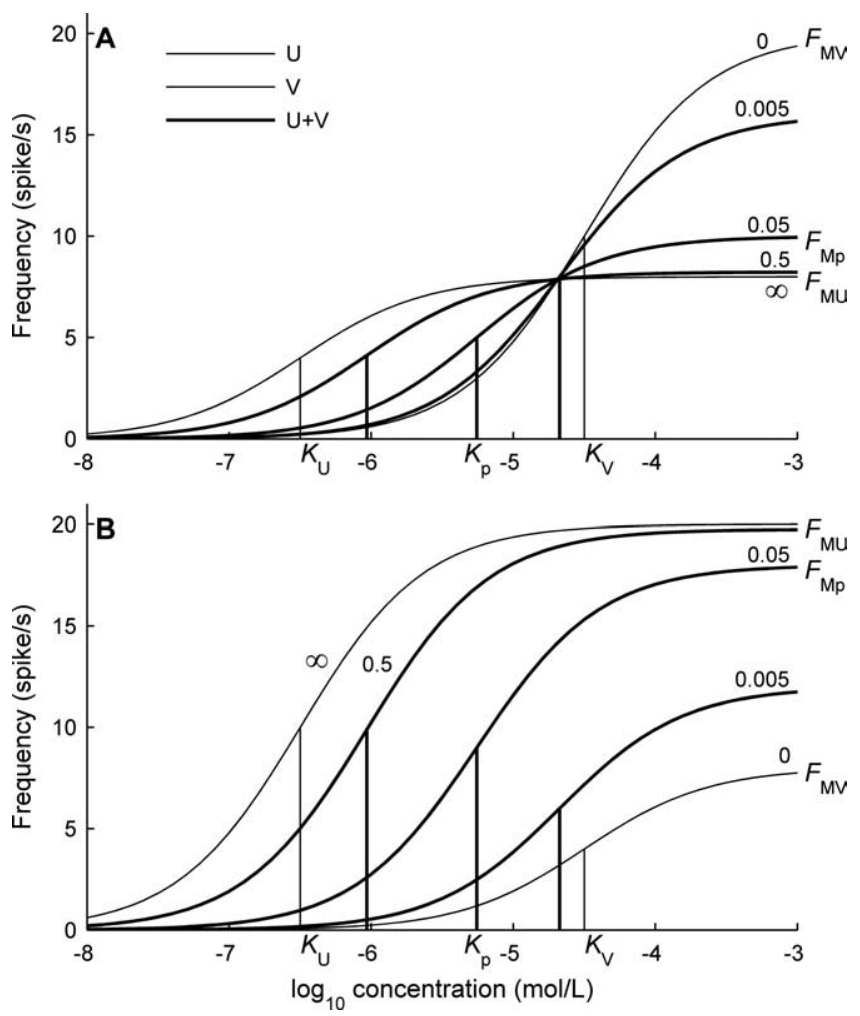

Figure 5. According to the model of syntopic (competitive only) interaction, the doseresponse curve for the mixture of two odorants is a sigmoid curve located between the doseresponse curves of the individual odorants. Horizontal axis, Concentration of the single odorants $(U, V)$ and total concentration $U+V$ of their mixture with ratio $r=U / V$ kept constant. The single-odorant curves (thin lines) in $\boldsymbol{A}$ and $\boldsymbol{B}$ preserve their $\mathrm{EC}_{50}$ values (vertical thin lines with $\boldsymbol{K}_{U}$ $<K_{\mathrm{V}}$ ) but differ in their maximal firing rate, so that they intersect (if $F_{\mathrm{MU}}<F_{\mathrm{MV}} ; \boldsymbol{A}$ ) or not (if $F_{\mathrm{MU}}$ $\left.>F_{M V} ; \boldsymbol{B}\right)$. Note that $F_{\mathrm{MU}}$ in $\boldsymbol{A}$ is equal to $F_{\mathrm{MV}}$ in $\boldsymbol{B}$, and $F_{\mathrm{MV}}$ in $\boldsymbol{A}$ is equal to $F_{\mathrm{MU}}$ in $\boldsymbol{B}$. In both cases, the $\mathrm{EC}_{50}$ values $K_{\mathrm{p}}$ (thick vertical lines) and maxima $F_{\mathrm{Mp}}$ for the mixture depend on $r$ (numbers on the right). For $r=0$ (respectively $\infty$ ), the mixture curve coincides with the curve of $V$ (respectively $U)$. In $A$, all curves intersect at the same point.

cacy results entirely from the second step (activation) of the odorant-OR interaction. In contrast, binding only without distinct activation would yield the same maximum for all odorants.

\section{Predictable and unpredictable responses to binary mixtures} The ORN response to a binary mixture $U+V$ can be calculated in case of competitive interaction between odorants $\mathrm{U}$ and $\mathrm{V}$ that bind to the same recognition site (syntopy) on the OR. Syntopy has been originally applied to the mixture problem under the assumption that the transduction cascade was triggered by the mere binding of an odorant to the OR (Beidler, 1962; Ennis, 1991; Getz and Akers, 1995). Such a single-step activation being unsatisfactory as shown above, we developed a model of syntopic interaction with two steps, binding then activation. In this model, the affinity of the odorants for the OR and their efficacy at activating the transduction cascade remain the same whether they are applied alone or in mixture. In empirical terms, syntopic interaction requires that the characteristics $\left(K_{\mathrm{U}}, F_{\mathrm{MU}}, K_{\mathrm{V}}, F_{\mathrm{MV}}\right)$ of odorants $\mathrm{U}$ and $\mathrm{V}$ and the Hill coefficient $n$ are not modified in the mixture. If these conditions were satisfied, the model presented here would make it possible to calculate the effect of any mixture on an ORN, provided the characteristics of each odorant-OR pair were known. Moreover, the ratio $r$ could play a role in quality coding by making the identification of a stimulus independent of its concentration (ratio coding) (SanchezMontañés and Pearce, 2006). Conversely, invalidation of the syntopic model would mean that the effect of a mixture is difficult to predict, if not unpredictable in practice, and would limit the effectiveness of ratio coding.

In accordance with the observation that agonist pairs, likely binding to the same site on the human OR1G1 receptor (G. Sanz, personal communication), may have suppressive effects (Sanz et al., 2005), we showed that in $47 \%$ (Table 3 ) of comparisons, the Hill function fitted to the experimental data was undistinguishable from the Hill function predicted by the syntopic model. It suggests that, in these cases, only competition between odorants was present, or at least that effects of a different nature were too weak to reach statistical significance. In the remaining comparisons, the observed Hill curves departed significantly from the predicted Hill curves in $\mathrm{EC}_{50}$, maximum, or both. Several explanations of the failure of the model to account for the mixture data were considered.

First, transport and deactivation of odorants in the mucus layer must be taken into account. Because of delays in removal, the odorant concentration in mucus grows to a higher level than in the air (Rospars et al., 2003), so that the odorant affinities for the receptors are not equal to the measured $\mathrm{EC}_{50}$ values. The fact that only apparent affinities are known does not affect the present discussion. However, differences in transport (Atanasova et al., 2005) or enzymatic deactivation of the odorants in the mucus might also change the ratio $r$ at the vicinity of the ORs with respect to the ratio in the air, the only one which is presently known. Such a change of $r$ would modify the predicted $\mathrm{EC}_{50}$ and maximum response. The fact that the same pairs of odorants $(\mathrm{ANI}+\mathrm{ISO}, \mathrm{CAM}+\mathrm{LIM}, \mathrm{LIM}+\mathrm{MEN})$ were found in the sets that obey the syntopic model and those that do not (Table 3 ), indicates that predictability would not be recovered by developing a more elaborate mucus model.

Second, antagonistic interactions between odorants and ORs are not likely in our experiments, because all odorants tested were agonists. This is a consequence of the protocol aiming at maximizing the number of recorded odorants per ORN. If an odorant was found to be inactive, it was not further tested in mixtures, which prevented observation of its possible antagonistic effect on other odorants (Araneda et al., 2000; Oka et al., 2004a,b; Sanz et al., 2005).

Finally, receptor and postreceptor mechanisms appear as the most promising explanations. They involve, for example, allosteric modulation of the OR, the effect of different OR conformations on distinct G-proteins, or direct effects on the transduction 
Table 3. Comparison of the observed characteristics (subscript " 0 ") of binary mixtures with those predicted (subscript " $\mathrm{p}^{\prime \prime}$ ) by the model of syntopic interaction

\begin{tabular}{|c|c|c|c|c|c|c|c|c|c|c|c|c|c|c|c|c|c|}
\hline \multicolumn{4}{|c|}{$\overline{\text { Sets }^{a}}$} & \multicolumn{2}{|c|}{ Sizes $^{b}$} & \multicolumn{6}{|c|}{ Comparison of $\mathrm{EC}_{50 \mathrm{~s}}(\log \mathrm{molar})^{\mathrm{c}}$} & \multicolumn{6}{|c|}{ Comparison of maxima (spikes/s) $^{d}$} \\
\hline ORN & U & V & $\log (r)$ & $N_{0}$ & $N_{\mathrm{p}}$ & $\log K_{0}$ & $\log K_{\mathrm{p}}$ & $\log K_{\mathrm{o}} / K_{\mathrm{p}}$ & $t_{\mathrm{K}}$ & $P_{\mathrm{K}}$ & $S_{K}$ & $F_{\text {Mo }}$ & $F_{\mathrm{Mp}}$ & $F_{\mathrm{Mo}}-F_{\mathrm{Mp}}$ & $t_{\mathrm{F}}$ & $P_{\mathrm{F}}$ & $S_{F}$ \\
\hline 15.4 & ANI & ISO & -0.199 & 7 & 7 & $-6.0 \pm 0.2$ & $-5.9 \pm 0.3$ & -0.10 & 0.57 & 0.71 & NS & $153 \pm 31$ & $150 \pm 19$ & 3.1 & 0.17 & 0.57 & \\
\hline 09.3 & CIT & LIL & 1.005 & 5 & 5 & $-8.4 \pm 1.5$ & $-7.8 \pm 0.5$ & -0.54 & 0.69 & 0.74 & NS & $12 \pm 6$ & $13 \pm 2$ & -1.1 & 0.31 & 0.62 & \\
\hline 37.6 & CAM & LIM & -1.304 & 6 & 6 & $-4.7 \pm 0.4$ & $-4.9 \pm 0.3$ & 0.20 & 0.82 & 0.78 & NS & $42 \pm 22$ & $33 \pm 12$ & 8.2 & 0.64 & 0.73 & \\
\hline 37.6 & LIM & MEN & 0.593 & 10 & 6 & $-5.9 \pm 0.2$ & $-5.8 \pm 0.2$ & -0.17 & 1.10 & 0.86 & NS & $49 \pm 5$ & $55 \pm 6$ & -5.8 & 1.44 & 0.91 & \\
\hline 37.6 & CAM & MEN & -0.711 & 10 & 7 & $-6.2 \pm 0.2$ & $-6.4 \pm 0.2$ & 0.16 & 1.14 & 0.86 & NS & $61 \pm 5$ & $56 \pm 6$ & 5.6 & 1.46 & 0.92 & \\
\hline 28.1 & LIM & LYR & 4.725 & 7 & 5 & $-5.0 \pm 0.2$ & $-5.1 \pm 0.2$ & 0.17 & 1.29 & 0.89 & NS & $100 \pm 25$ & $75 \pm 17$ & 24.1 & 1.54 & 0.92 & \\
\hline 27.1 & EVA & LYR & -0.419 & 5 & 5 & $-9.4 \pm 0.2$ & $-9.6 \pm 0.2$ & 0.22 & 1.44 & 0.91 & NS & $31 \pm 12$ & $16 \pm 17$ & 15.8 & 1.51 & 0.92 & \\
\hline 39.1 & ANI & ISO & -0.199 & 6 & 6 & $-4.3 \pm 0.4$ & $-4.3 \pm 0.2$ & -0.02 & 0.07 & 0.53 & NS & $54 \pm 26$ & $90 \pm 15$ & -35.5 & 2.32 & 0.98 & J \\
\hline 40.2 & LIM & MEN & 0.593 & 7 & 5 & $-5.2 \pm 0.1$ & $-5.2 \pm 0.2$ & -0.03 & 0.28 & 0.61 & NS & $57 \pm 8$ & $44 \pm 8$ & 12.8 & 2.25 & 0.98 & $S$ \\
\hline 17.1 & LYR & MEN & -4.132 & 7 & 7 & $-5.9 \pm 0.4$ & $-6.2 \pm 0.3$ & 0.34 & 1.28 & 0.89 & NS & $32 \pm 10$ & $7 \pm 2$ & 25.0 & 4.98 & 1.00 & $S$ \\
\hline 28.1 & LYR & MEN & -4.132 & 5 & 5 & $-7.0 \pm 0.3$ & $-7.4 \pm 0.1$ & 0.33 & 2.31 & 0.98 & $S$ & $99 \pm 23$ & $112 \pm 7$ & -13.0 & 1.06 & 0.84 & \\
\hline 15.4 & ANI & MAK & -0.038 & 11 & 6 & $-6.7 \pm 0.2$ & $-6.3 \pm 0.3$ & -0.43 & 2.24 & 0.98 & $S$ & $192 \pm 20$ & $203 \pm 49$ & -11.4 & 0.42 & 0.66 & \\
\hline 17.3 & CAM & CIT & 0.299 & 8 & 5 & $-7.0 \pm 0.2$ & $-6.8 \pm 0.1$ & -0.24 & 1.99 & 0.96 & $S$ & $21 \pm 5$ & $36 \pm 6$ & -14.7 & 3.77 & 1.00 & $S$ \\
\hline 17.3 & CAM & LIM & -1.304 & 7 & 7 & $-5.8 \pm 0.2$ & $-6.4 \pm 0.2$ & 0.59 & 4.20 & 1.00 & $S$ & $29 \pm 6$ & $16 \pm 3$ & 13.7 & 4.40 & 1.00 & $S$ \\
\hline 17.1 & LIM & MEN & 0.593 & 7 & 7 & $-5.8 \pm 0.1$ & $-5.2 \pm 0.1$ & -0.62 & 7.66 & 1.00 & $S$ & $36 \pm 3$ & $48 \pm 5$ & -12.7 & 4.25 & 1.00 & $S$ \\
\hline
\end{tabular}

${ }^{a}$ Ratio $U_{0} / V_{0}$ of molarities of saturating vapors of odorants $U$ and $V$ (see Table 1).

${ }^{b}$ Number $N_{\mathrm{p}}$ of points of predicted curve is the smallest of the number of stimulations with $\mathrm{U}\left(N_{\mathrm{U}}\right)$ and $\mathrm{V}\left(N_{\mathrm{V}}\right)$.

'Predicted $\mathrm{EC}_{50}$ values $\left(\log K_{\mathrm{p}} \pm \Delta \log K_{\mathrm{p}}\right.$ ) given by Equation 2 and supplemental Equation A24 (available at www.jneurosci.org as supplemental material). Difference $\log K_{0}-\log K_{\mathrm{p}}$, with observed $\mathrm{EC}_{50}\left(\right.$ log $K_{0} \pm \Delta \log K_{0}$ ) significantly different from 0 ( $S$ in column $S_{K}$ ) at significance level $5 \%$, when $P$ value $P_{K}$ of $t$ test $t_{K}$ with $d f$ degrees of freedom ( $\left.d f=N_{o}+N_{p}-2\right)$ greater than 0.95 . Not significant (NS) otherwise.

${ }^{d}$ Predicted asymptotic firing rates $\left(F_{\mathrm{Mp}} \pm \Delta F_{\mathrm{Mp}}\right)$ given by Equation 3 and supplemental Equation A29 (available at www.jneurosci.org as supplemental material). Difference $F_{\mathrm{Mo}}-F_{\mathrm{Mp}}$, with observed maximum firing rate $\left(F_{\mathrm{Mo}} \pm \Delta F_{\mathrm{Mo}}\right)$, tested by $t$ test at level $5 \%$, with $t_{F,} P_{F}$ and $S_{F}$ as for $\mathrm{EC}_{50}$.
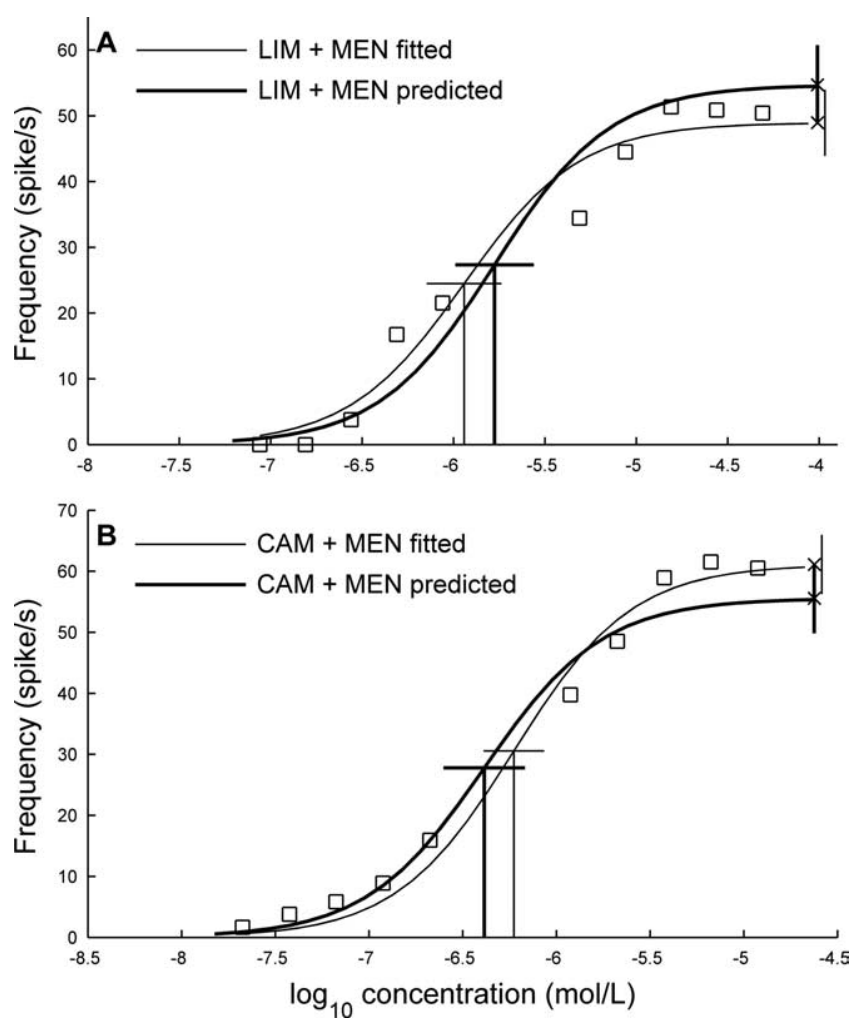

Figure 6. Examples of responses to mixtures in agreement with those predicted by the model of syntopic interaction. $A$, ORN 37.6 LIM + MEN as in Figure 2A. B, ORN 37.6 CAM + MEN as in Figure $2 B$. Observed Hill curve (thin line) fitted to experimental mixture points (squares), with $\mathrm{EC}_{50}\left(\log K_{0} \pm \Delta \log K_{0}\right)$ and maximum $\left(F_{\mathrm{M}_{0}} \pm \Delta F_{\mathrm{M}_{0}}\right)$ represented as in Figure 2. Predicted Hill curve (thick line) calculated from the syntopic model with $\mathrm{EC}_{50}\left(\log K_{\mathrm{p}} \pm \Delta \log K_{\mathrm{p}}\right)$ and $\operatorname{maximum}\left(F_{\mathrm{Mp}} \pm \Delta \log K_{\mathrm{p}}\right)$.

cascade. Although our experiments do not permit to choose between these hypotheses, allosterism is worth further attention. Allosteric modulation is common in G-protein-coupled receptors, including the rhodopsine family to which ORs belong (Jensen and Spaldong, 2004; Gao and Jacobson, 2006), and is
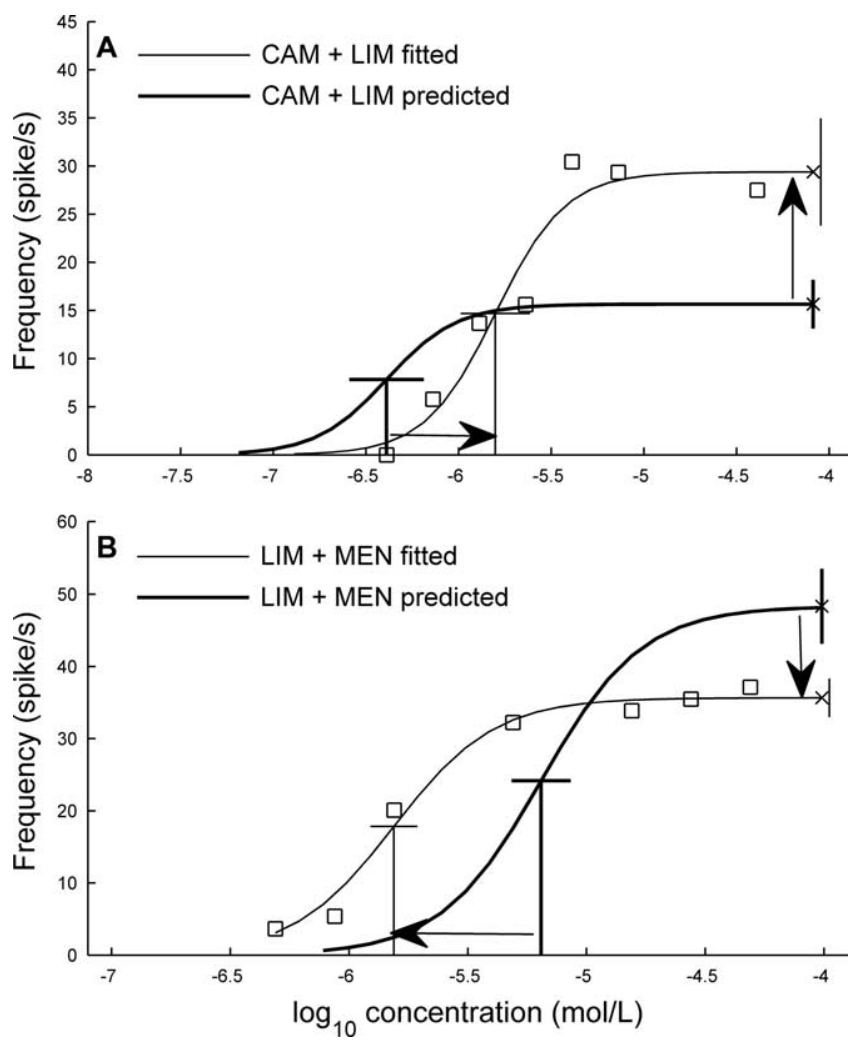

Figure 7. Examples of responses to mixtures (thin lines) diverging from those expected from purely competitive (syntopic) interactions (thick lines). Same representation as in Figure 6, with arrows showing noncompetitive effects. $A, 0$ RN 17.3 as in Figure $2 C$, where partial suppression results from a decrease in apparent affinity (horizontal arrow) and an increase in efficacy (vertical arrow) with respect to the reference case. $\boldsymbol{B}$, ORN 17.1 as in Figure 2D, where partial synergy results from an increase in apparent affinity and decrease in efficacy (arrows).

consistent with the effects reported here. The observation that the shifts in $\mathrm{EC}_{50}$ values and maxima have no preferred direction suggests that these quantities can be increased or decreased with equal probabilities. It implies, in the framework of the phenomenological model used here, that one of the components of a 
mixture could modify the affinity (i.e., binding step) and/or the efficacy (i.e., activation step) of the OR for the other component, and both steps could be modulated independently. Four possible cases follow according to whether the allosteric agonist or antagonist effects on $\mathrm{EC}_{50}$ and on maximum are the same or opposite. The two cases with opposite actions would explain why the effects are not the same at all concentrations, as illustrated by the observation of partial inhibitory (Fig. 2C) and synergistic (Fig. 2D) mixture interactions.

\section{Consequences for the neural coding of natural odors}

Whatever the receptor or postreceptor mechanisms involved in the noncompetitive modification observed, they have significant consequences for the neural coding of natural odors and therefore for understanding the formation of an olfactory percept. Indeed, let us assume that the probability of a given odorant $U$ does not modify the receptor and postreceptor properties of an ORN for another odorant $\mathrm{V}$ is $p$, for example 0.7 . The probability that mixture $\mathrm{U}+\mathrm{V}$ does not modify the properties of the ORN is then $p^{2}=0.7 \times 0.7=0.49$, which is close to the observed $47 \%$ (Table 3 ). If the number of components increases, the probability that no property is modified decreases. For a ternary mixture, it becomes $p^{3}=0.34$, etc. The relatively large value of probability $p$ suggests that noncompetitive effects involving unrelated agonists are quite common.

An analogous reasoning applies to a population of different ORs born by a population of diverse ORNs. Therefore, the probability that no OR is modified by the mixture is very low. It follows that, in general, the image generated by a mixture is not predictable from the known properties of its individual components. Although large deviations are expected to be rare (Davison and Katz, 2007) because of the opposite effects mentioned above, noncompetitive modulation opens a new combinatorial dimension, which may add much complexity to the effect of an odor stimulus and contribute to the emergence of new perceptual qualities not present in each component (Jinks et al., 2001; Wiltrout et al., 2003).

\section{References}

Aickin M, Gensler H (1996) Adjusting for multiple testing when reporting research results: the Bonferroni vs Holm methods. Am J Public Health $86: 726-728$.

Araneda RC, Kini AD, Firestein S (2000) The molecular receptive range of an odorant receptor. Nat Neurosci 3:1248-1255.

Araneda RC, Peterlin Z, Zhang X, Chesler A, Firestein S (2004) A pharmacological profile of the aldehyde receptor repertoire in rat olfactory epithelium. J Physiol (Lond) 555:743-756.

Atanasova B, Thomas-Danguin T, Chabanet C, Langlois D, Nicklaus S, Etiévant $P$ (2005) Perceptual interactions in odour mixtures: odour quality in binary mixtures of woody and fruity wine odorants. Chem Senses 30:209-217.

Beidler LM (1962) Taste receptor stimulation. In: Progress in biophysics and biophysical chemistry (Butler JAV, Huxley HE, Zirkle RE, eds), pp 12:107-151. Oxford: Pergamon.

Buck L, Axel R (1991) A novel multigene family may encode odorant receptors. A molecular basis for odor recognition. Cell 65:16-29.

Davison IG, Katz LC (2007) Sparse and selective odor coding by mitral/ tufted neurons in the main olfactory bulb. J Neurosci 27:2091-2101.

Derby CD, Hutson M, Livermore BA, Lynch WH (1996) Generalization among related complex odorant mixtures and their components: analysis of olfactory perception in the spiny lobster. Physiol Behav 60:87-95.

Del Castillo J, Katz B (1957) Interaction at end-plate receptors between different choline derivatives. Proc R Soc Lond B Biol Sci 146:369-381.

Duchamp-Viret P, Chaput MA, Duchamp A (1989) Amplifying role of convergence in olfactory system. A comparative study of receptor cell and second order neuron sensitivities. J Neurophysiol 61:1085-1094.

Duchamp-Viret P, Duchamp A, Chaput MA (2000) Peripheral odor coding in the rat and frog: quality and intensity specification. J Neurosci 20:2383-2390.

Duchamp-Viret P, Duchamp A, Chaput MA (2003) Single olfactory sensory neurons simultaneously integrate the components of an odour mixture. Eur J Neurosci 18:2690-2696.

Ennis DM (1991) Molecular mixture models based on competitive and non-competitive agonism. Chem Senses 16:1-17.

Firestein S, Picco C, Menini A (1993) The relation between stimulus and response in olfactory receptor cells of the tiger salamander. J Physiol (Lond) 468:1-10.

Gao ZG, Jacobson KA (2006) Allosterism in membrane receptors. Drug Discov Today 11:191-202.

Getz WM, Akers RP (1995) Partitioning non-linearities in the response of honey bee olfactory receptor neurons to binary odors. BioSystems 34:27-40.

Gleeson RA, Ache BW (1985) Amino acid suppression of taurine-sensitive chemosensory neurons. Brain Res 335:99-107.

Godfrey PA, Malnic B, Buck LB (2004) The mouse olfactory receptor gene family. Proc Natl Acad Sci USA 101:2156-2161.

Grosmaître X, Vassalli A, Mombaerts P, Shepherd GM, Ma M (2006) Odorant responses of olfactory sensory neurons expressing the odorant receptor MOR23: a patch clamp analysis in gene-targeted mice. Proc Natl Acad Sci USA 103:1970-1975.

Jensen AA, Spaldong TA (2004) Allosteric modulation of G-protein coupled receptors. Eur J Pharm Sci 21:407-420.

Jinks A, Laing DG, Hutchinson I (2001) A psychophysical study of the relationship between temporal processing in odor mixtures and transduction pathways. Brain Res Cogn Brain Res 12:273-288.

Kang J, Caprio J (1997) In vivo responses of single olfactory receptor neurons of channel catfish to binary mixtures of amino acids. J Neurophysiol 77:1-8.

Laing DG, Francis GW (1989) The capacity of humans to identify odors in mixtures. Physiol Behav 46:809-814.

Laing DG, Panhuber H, Slotnik BM (1989) Odor masking in the rat. Physiol Behav 45:689-694.

Larson HJ (1973) Introduction to the theory of statistics. New York: Wiley. Laska M, Hudson R (1992) Ability to discriminate between related odor mixtures. Chem Senses 17:403-415.

Malnic B, Godfrey PA, Buck LB (2004) The human olfactory receptor gene family. Proc Natl Acad Sci USA 101:2584-2589.

Neubig RR, Spedding M, Kenakin T, Christopoulos A (2003) International union of pharmacology committee on receptor nomenclature and drug classification. XXXVIII. Update on terms and symbols in quantitative pharmacology. Pharmacol Rev 55:597-606.

Oka Y, Nakamura A, Watanabe H, Touhara K (2004a) An odorant derivative as an antagonist for an olfactory receptor. Chem Senses 29:815-822.

Oka Y, Omura M, Kataoka H, Touhara K (2004b) Olfactory receptor antagonism between odorants. EMBO J 23:120-126.

Rang HP (2006) The receptor concept: pharmacology's big idea. Br J Pharmacol 147:S9-S16.

Reisert J, Matthews HR (1999) Adaptation of the odour-induced response in frog olfactory receptor cells. J Physiol (Lond) 519:801-813.

Rospars JP, Lansky P, Tuckwell HC, Vermeulen A (1996) Coding of odor intensity in a steady-state deterministic model of an olfactory receptor neuron. J Comput Neurosci 3:51-72.

Rospars JP, Lansky P, Duchamp A, Duchamp-Viret P (2003) Relation between stimulus and response in frog olfactory receptor neurons in vivo. Eur J Neurosci 18:1135-1154.

Sanchez-Montañés M, Pearce T (2006) Ratio coding of odour mixtures in olfactory receptor neurons. ECRO Abstr 17:193-194.

Sanz G, Schlegel C, Pernollet JC, Briand L (2005) Comparison of odorant specificity of two human olfactory receptors from different phylogenetic classes and evidence for antagonism. Chem Senses 30:69-80.

Serizawa S, Miyamichi K, Nakatani H, Suzuki M, Saito M, Yoshihara Y, Sakano H (2003) Negative feedback regulation ensures the one receptor-one olfactory neuron rule in mouse. Science 302:2088-2094.

Vigouroux M, Viret P, Duchamp A (1988) A wide concentration range olfactometer for delivery of short reproducible odor pulses. J Neurosci Methods 24:57-63.

Wiltrout C, Dogra S, Linster C (2003) Configurational and nonconfigurational interactions between odorants in binary mixtures. Behav Neurosci $117: 236-245$ 